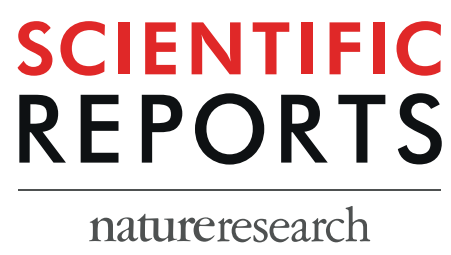

\title{
OPEN Antifungal drug susceptibility, molecular basis of resistance to echinocandins and molecular epidemiology of fluconazole resistance among clinical Candida glabrata isolates in Kuwait
}

\begin{abstract}
Zahraa F. Al-Baqsami, Suhail Ahmad* \& Ziauddin Khan
Candida glabrata readily develops resistance to echinocandins. Identification, antifungal susceptibility testing (AST) and resistance mechanism to echinocandins among C. glabrata was determined in Kuwait. C. glabrata isolates $(n=75)$ were tested by Vitek2, multiplex PCR and/or PCR-sequencing of rDNA. AST to fluconazole, caspofungin, micafungin and amphotericin $B$ was determined by Etest and to micafungin by broth microdilution (BMD). Mutations in hotspot-1/hotspot-2 of FKS1/FKS2 and ERG11 were detected by PCR-sequencing. All isolates were identified as C. glabrata sensu stricto. Seventy isolates were susceptible and five were resistant to micafungin by Etest and BMD (essential agreement, 93\%; categorical agreement, $100 \%$ ). Three micafungin-resistant isolates were resistant and two were susceptible dose-dependent to caspofungin. Four and one micafungin-resistant isolate contained S663P and $\triangle 659 \mathrm{~F}$ mutation, respectively, in hotspot- 1 of $F K S 2$. Micafungin-resistant isolates were genotypically distinct strains. Only one of 36 fluconazole-resistant isolate contained nonsynonymous ERG11 mutations. Thirty-four of 36 fluconazole-resistant isolates were genotypically distinct strains. Our data show that micafungin susceptibility reliably identifies echinocandin-resistant isolates and may serve as a surrogate marker for predicting susceptibility/resistance of $C$. glabrata to caspofungin. All micafungin-resistant isolates also harbored a nonsynonymous/deletion mutation in hotspot-1 of FKS2. Fingerprinting data showed that echinocandin/fluconazole resistance development in C. glabrata is not clonal.
\end{abstract}

Candida spp. are the fourth most common cause of bloodstream infections in hospitalized patients and third common cause of central-line associated invasive infections among intensive care unit (ICU) patients ${ }^{1-3}$. Epidemiological studies have shown that $>90 \%$ of invasive infections are caused by only five species/species complexes, namely Candida albicans, Candida glabrata, Candida tropicalis, Candida parapsilosis, and Candida kruse $^{1-3}$. Although C. albicans is most commonly isolated from patients with invasive infections and is also the most pathogenic species, infections by non-albicans Candida species have increased dramatically in recent years ${ }^{3-}$ ${ }^{8}$. The prophylactic and/or empirical treatment of susceptible immunocompromised/immunosuppressed patients has also resulted in increased prevalence of infections caused by drug-resistant and multidrug-resistant Candida species $^{9-14}$. C. glabrata has now emerged as the second or third most frequently isolated Candida species from patients, particularly from critically ill older ( $>65$ years) patients, with bloodstream and other invasive infections as well as those with vulvovaginal and oral infections, $s^{6,14-18}$.

C. glabrata, a haploid fungal pathogen, is intrinsically less susceptible to azole antifungal drugs and invasive infections are associated with high $(\sim 50 \%)$ mortality rates, particularly in immunocompromised elderly patients requiring major surgery and neutropenic patients ${ }^{2,5-7,19,20}$. Due to reduced susceptibility of some Candida spp. to triazoles, echinocandins were recently promoted as first-line agents for the treatment of invasive Candida 


\begin{tabular}{|l|l|l|l|l|l|l|l|l|l|l|l|l|l|l|l|l|l|l|l|l|l|l|l|l|l|l|}
\hline Antifungal & \multicolumn{10}{|c|}{ Number of isolates with indicated minimum inhitory concentration (MIC) in $\boldsymbol{\mu g} / \mathbf{m l}$} \\
\hline drug & $\leq 0.008$ & 0.012 & 0.016 & 0.023 & 0.032 & 0.047 & 0.064 & 0.094 & 0.125 & 0.19 & 0.25 & 0.38 & 0.5 & 0.75 & 1 & 1.5 & 2 & 3 & 4 & 6 & 8 & 12 & 16 & 24 & $\geq 32$ \\
\hline Micafungin & 13 & 16 & $\underline{28}$ & 11 & 2 & 0 & 0 & 0 & $\mathbf{4}$ & 0 & $\mathbf{1}$ & 0 & 0 & 0 & 0 & 0 & 0 & 0 & 0 & 0 & 0 & 0 & 0 & 0 & 0 \\
\hline Caspofungin & 1 & 1 & 1 & 3 & 4 & 2 & 3 & 13 & 7 & 12 & $\underline{14}$ & 10 & $\mathbf{1}$ & $\mathbf{3}$ & 0 & 0 & 0 & 0 & 0 & 0 & 0 & 0 & 0 & 0 & 0 \\
\hline Fluconazole & 0 & 0 & 0 & 0 & 0 & 0 & 0 & 0 & 1 & 0 & 0 & 1 & 0 & 2 & 0 & 2 & 0 & 2 & 7 & 4 & 6 & 5 & 6 & 3 & $\underline{36}$ \\
\hline Amphotericin B & 1 & 1 & 1 & 2 & 2 & 6 & 7 & 10 & 9 & $\underline{14}$ & 6 & 6 & 2 & 1 & 2 & 2 & 1 & 1 & 1 & 0 & 0 & 0 & 0 & 0 & 0 \\
\hline
\end{tabular}

Table 1. Antifungal susceptibility patterns of clinical C. glabrata isolates against various antifungal agents by Etest. Isolates with MICs indicative of resistance or reduced susceptibility to antifungal drugs are highlighted in bold. The modal values are underlined.

infections ${ }^{3}$. However, resistance to echinocandins in Candida spp. has also appeared in recent years with the highest rate occurring among C. glabrata and breakthrough invasive C. glabrata infections have been reported in patients on micafungin therapy $y^{14-16,21-24}$. Resistance to polyenes is also being reported with increasing frequency in clinical C. glabrata isolates ${ }^{25-29}$ and a multidrug-resistant phenotype (resistant to azoles and echinocandins) occurring in ICU and non-ICU settings has also been described in recent years ${ }^{30,31}$.

Acquired resistance to echinocandins in C. glabrata makes antifungal susceptibility testing mandatory to guide therapeutic decisions. Echinocandins inhibit cell wall synthesis by binding to their multi-subunit target, 1,3-3-D-glucan synthase complex, encoded by FKS1, FKS2 and FKS3 in C. glabrata and other Candida spe$\operatorname{cies}^{5,19,20}$. Studies have shown that clinical echinocandin resistance in C. glabrata is due to amino acid substitutions in the hotspot- 1 and hotspot- 2 regions of the two subunits of 1,3- $\beta$-D-glucan synthase complex encoded by FKS1 and FKS2 genes ${ }^{5,19,20}$. Detection of mutations in FKS genes is now considered as the most accurate method to predict treatment failure even when the patients lack usual risk factors for echinocandin resistance development $^{20,22-24,32}$. This study determined antifungal susceptibility of clinical C. glabrata isolates and the molecular basis of resistance to echinocandins by PCR-sequencing of hotspot-1 and hotspot-2 regions of FKS1 and FKS2 genes. The ERG11 was also sequenced from fluconazole-resistant C. glabrata isolates. Fingerprinting was carried out to determine whether drug-resistant C. glabrata isolates were clonally related.

\section{Results}

Phenotypic and molecular identification of clinical C. glabrata isolates. All 75 isolates initially identified as C. glabrata sensu lato by Vitek2 produced purple (mauve) color on CHROMagar Candida and yielded an amplicon of $\sim 212 \mathrm{bp}$ 'in PCR assay which are characteristic of C. glabrata sensu stricto strains. PCRsequencing of the internally transcribed spacer (ITS) region (including ITS-1-5.8 S rRNA-ITS-2) of rDNA also identified all 51 selected isolates as C. glabrata sensu stricto as they exhibited maximum ( $>99 \%)$ identity with reference C. glabrata strains ATCC 90030 or CBS138. The ITS region sequence data also showed genotypic heterogeneity as 23 different haplotypes (ITSH1 to ITSH23) were detected among 51 C. glabrata isolates (16 isolates yielded unique haplotypes while the remaining seven haplotypes were shared among 35 isolates in seven clusters) from Kuwait.

Antifungal susceptibility profile of $C$. glabrata isolates. The antifungal susceptibility testing (AST) data against micafungin, caspofungin, fluconazole, and amphotericin B by Etest are presented in Table 1. According to EUCAST clinical breakpoints, 70 of 75 (93.3\%) isolates were susceptible to micafungin with a modal minimum inhibitory concentration (MIC) value of $0.016 \mu \mathrm{g} / \mathrm{ml}$ while five isolates exhibited resistance as they showed an MIC value $>0.125 \mu \mathrm{g} / \mathrm{ml}$. The MIC distribution for all susceptible isolates was within two twofold dilution steps surrounding the modal MIC. Interestingly, only three of five micafungin-resistant isolates and one micafungin-susceptible isolate were resistant (MIC $\geq 0.5 \mu \mathrm{g} / \mathrm{ml}$ ) to caspofungin by Etest (Table 1 ). Of the remaining 71 isolates, 24 isolates were in the intermediate range (MIC of $>0.25 \mu \mathrm{g} / \mathrm{ml}$ but $<0.5 \mu \mathrm{g} / \mathrm{ml}$ ) (including two micafungin-resistant isolates) while 47 isolates were susceptible to caspofungin. For fluconazole, 39 of 75 (52\%) isolates were susceptible dose-dependent $(\mathrm{MIC}=<32 \mu \mathrm{g} / \mathrm{ml})$ while the remaining $36(48 \%)$ isolates yielded MIC values $>32 \mu \mathrm{g} / \mathrm{ml}$ and were scored as resistant (Table 1). For amphotericin B, 70 of 75 (93.3\%) isolates yielded MIC values $\leq 1 \mu \mathrm{g} / \mathrm{ml}$ and were categorized as susceptible (or wild-type) while five (6.7\%) isolates were resistant (or non-wild-type) as they yielded MIC values $>1 \mu \mathrm{g} / \mathrm{ml}$ (Table 1 ).

The AST against micafungin was also carried out by broth microdilution (BMD) method. All five C. glabrata isolates resistant to micafungin by Etest were also resistant (MIC $>0.25 \mu \mathrm{g} / \mathrm{ml}$ ) by BMD method while the remaining 70 isolates were susceptible (MIC $<0.03 \mu \mathrm{g} / \mathrm{ml}$ ). The modal MIC value was $0.007 \mu \mathrm{g} / \mathrm{ml}$ and the MIC distribution for all susceptible isolates was within two twofold dilution steps surrounding the modal MIC. Interestingly, when CLSI clinical breakpoints were considered, 70 isolates were micafungin-susceptible, four were in the intermediate range (but resistant according to EUCAST breakpoints) and one isolate was micafungin-resistant. The correlation between MIC values obtained by Etest and BMD method are presented in Table 2. The data showed that $70(93.3 \%)$ isolates exhibited excellent essential agreement between the two methods as they yielded MIC values that were within 2-fold dilution difference. The remaining five isolates yielded poor essential agreement as they yielded MIC values that differed by $>2$-fold dilution difference by the two methods. However, the categorical agreement between the two methods was perfect as all five micafungin-resistant isolates by Etest were also resistant to micafungin by BMD and the remaining 70 isolates were susceptible to micafungin by both Etest 


\begin{tabular}{|c|c|c|c|c|c|c|c|c|c|c|}
\hline & & \multicolumn{9}{|c|}{ EUCAST MIC $(\mu \mathrm{g} / \mathrm{ml})$} \\
\hline & & $\leq \mathbf{0 . 0 0 3}$ & 0.007 & 0.015 & 0.03 & 0.06 & 0.25 & 1 & 2 & Total \\
\hline \multirow{10}{*}{ Etest MIC $(\mu \mathrm{g} / \mathrm{ml})$} & 0.003 & 1 & & & & & & & & 1 \\
\hline & 0.007 & 5 & 7 & & & & & & & 12 \\
\hline & 0.015 & 5 & 31 & 6 & 2 & & & & & 44 \\
\hline & 0.03 & $1^{*}$ & 12 & & & & & & & 13 \\
\hline & 0.06 & & & & & & & & & 0 \\
\hline & 0.125 & & & & & & & $1^{*}$ & $3 *$ & 4 \\
\hline & 0.25 & & & & & & 1 & & & 1 \\
\hline & 1 & & & & & & & & & \\
\hline & 2 & & & & & & & & & \\
\hline & Total & 12 & 50 & 6 & 2 & 0 & 1 & 1 & 3 & 75 \\
\hline
\end{tabular}

Table 2. Correlation between MIC values obtained by Etest and EUCAST broth microdilution (BMD) method during in vitro susceptibility testing of 75 C. glabrata isolates against micafungin. Etest MIC $(\mu \mathrm{g} / \mathrm{ml})$. *Isolates with MIC values that differed by $>2$ fold dilution between Etest and EUCAST BMD method. Micafunginresistant isolates are shown in bold.

\begin{tabular}{|c|c|c|c|c|c|c|c|c|c|c|c|}
\hline \multirow{2}{*}{$\begin{array}{l}\text { Patient } \\
\text { no. }\end{array}$} & \multicolumn{2}{|c|}{ Patient's details } & \multirow[b]{2}{*}{$\begin{array}{l}\text { Underlying } \\
\text { condition }\end{array}$} & \multirow{2}{*}{$\begin{array}{l}\text { CFG } \\
\text { treatment } \\
\text { duration }\end{array}$} & \multirow[b]{2}{*}{ Clinical source } & \multirow[b]{2}{*}{ Isolate no. } & \multicolumn{4}{|c|}{ Etest MIC $(\mu \mathrm{g} / \mathrm{ml})$ for } & \multirow{2}{*}{$\begin{array}{l}\text { Mutation } \\
\text { in } \\
\text { hotspot-1 } \\
\text { of } F K S 2\end{array}$} \\
\hline & Gender & Age & & & & & MFG & CFG & AMB & FLU & \\
\hline 1 & Female & 44 years & AML & 14 days & Urine & Kw164/15 & 0.125 & 0.75 & 0.38 & 64 & S663P \\
\hline 2 & Male & NA & NA & NA & Tracheal secretion & Kw3646/15 & 0.125 & 0.38 & 0.19 & 12 & S663P \\
\hline 3 & Male & 49 years & $60 \%$ burns & 14 days & Urine & Kw458/16 & 0.25 & 0.38 & 0.38 & 8 & $\Delta \mathrm{F} 659$ \\
\hline 4 & Female & 83 years & KTR & 14 days & Urine & Kw3554/16 & 0.125 & 0.75 & 0.094 & 4 & S663P \\
\hline 5 & Female & 74 years & CKD & 14 days & Urine & Kw2138/17 & 0.125 & 0.75 & 0.094 & 24 & S663P \\
\hline
\end{tabular}

Table 3. Patient's characteristics, clinical source, antifungal drug susceptibility profile and nonsynonymous/ deletion mutations in hotspot-1 of FKS2 among five micafungin-resistant C. glabrata isolates. MIC, minimum inhibitory concentration; CFG, caspofungin; MFG, micafungin; AMB, amphotericin B; FLU, fluconazole; AML, acute myeloid leukemia; KTR, kidney transplant recipient; CKD, chronic kidney disease; NA, not available. MIC values indicative of resistance to antifungal drugs are shown in bold.

and BMD method. Interestingly four of five micafungin-resistant isolates exhibited higher MIC values by BMD method (Table 2).

Detection of mutations in hotspot-1 and hotspot-2 of $F K S 1$ and $F K S 2$ genes. PCR amplification with CgFKS-1F + CgFKS-1R primers yielded an amplicon of $\sim 560$ bp from all 75 C. glabrata isolates. Similarly, PCR amplification with CgFKS-2F + CgFKS-2R primers yielded an amplicon of $~ 538$ bp from all 75 isolates. The purified amplicons were sequenced with gene and region-specific primers as detailed in 'Materials and Methods'. Although few synonymous mutations were detected, the translated DNA (amino acid) sequence data for hotspot-1 and hotspot- 2 of FKS1 and hotspot-2 of FKS2 from all 75 isolates were identical (wild-type) to the sequence from reference C. glabrata strain ATCC90030. However, sequence data for hotspot-1 of FKS2 from only 70 isolates were wild-type while four isolates contained a nonsynonymous (S663P) mutation and one isolate contained a three nucleotide deletion (corresponding to codon $\mathrm{F} 659)(\Delta \mathrm{F} 659)$. Interestingly, all 70 isolates with wild-type sequence for hotspot-1 of FKS2 were susceptible to micafungin while four isolates with S663P mutation and one isolate with $\Delta$ F659 mutation were resistant to micafungin (Table 3 and Supplementary Table S1). One to five synonymous mutations were also detected within the FKS2 gene fragment flanking hotspot-1 region in all micafungin-resistant and many micafungin-susceptible isolates.

The results of AST for the four antifungal drugs and mutations in FKS genes are summarized in Table 3. Only three (Kw164/15, Kw3554/16 and Kw2138/17) micafungin-resistant isolates with mutations in hotspot-1 of FKS2 exhibited cross-resistance to caspofungin while the remaining two isolates (Kw3646/15 and Kw458/16) with mutations in hotspot- 1 of FKS2 exhibited intermediate susceptibility (MIC $=0.38 \mu \mathrm{g} / \mathrm{ml}$ ) to caspofungin by Etest (Table 3). Furthermore, one caspofungin-resistant isolate (Kw330/15) by Etest was susceptible to micafungin by both Etest and BMD method and contained wild-type sequences of hotspot-1 and hotspot- 2 of FKS1 and FKS2. Only one of five micafungin resistant C. glabrata isolate (Kw164/15) exhibited cross resistance to fluconazole and thus exhibited multidrug-resistant phenotype while all five isolates were susceptible (wild-type) to amphotericin $\mathrm{B}(\mathrm{MIC}<1 \mu \mathrm{g} / \mathrm{ml})$ (Table 3). Clinical details and history of previous exposure to echinocandins were available for four patients yielding micafungin-resistant C. glabrata isolates with mutations at F659 or S663 in hotspot-1 of FKS2. Three patients were females including two elderly patients ( $\geq 74$ years). Four patients had received caspofungin for 14 days as treatment or prophylaxis and C. glabrata strains were isolated from urine samples from all four patients (Table 3). The information regarding the isolation of $C$. glabrata from invasive sites from these four patients and the outcome were not available. 


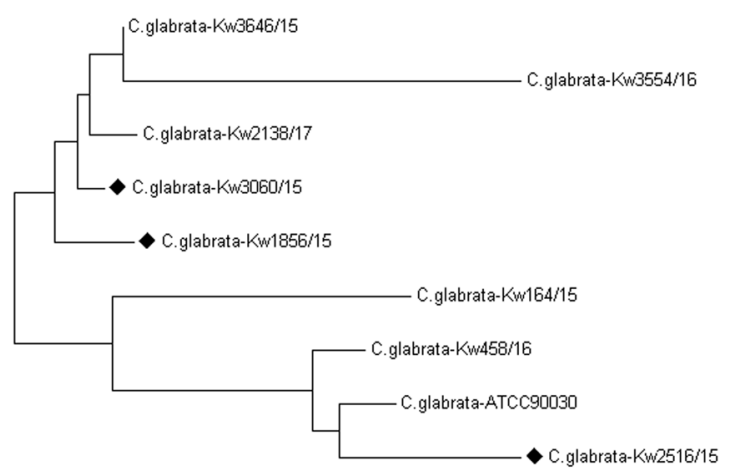

$\longdiv { 0 . 0 0 0 5 }$

Figure 1. Neighbor-Joining phylogenetic tree based on Maximum Composite Likelihood of DNA sequence data for hotspot-1 and hotspot- 2 of FKS1 and FKS2 genes together with ITS region of rDNA for five micafungin-resistant C. glabrata isolates. Three micafungin-susceptible isolates $(\boldsymbol{\nabla})$ and reference strain (ATCC90030) of C. glabrata were included for comparison purpose.

The phylogenetic relationship among five micafungin-resistant isolates with mutations in hotspot-1 of FKS2, three selected micafungin-susceptible isolates and reference C. glabrata strain ATCC 90030 was also determined from concatenated DNA sequence data for hotspot-1 and hotspot- 2 of FKS1 and FKS2 and the ITS region of rDNA. The data showed that all five micafungin-resistant isolates were genotypically distinct strains (Fig. 1).

Analysis of ERG11 gene sequences of C. glabrata isolates. The ERG11 gene was amplified as two overlapping fragments and both strands were sequenced from all 36 fluconazole-resistant and three susceptible dose-dependent C. glabrata strains. Although few synonymous mutations within the coding region of ERG11 and/or insertion/deletion/single nucleotide polymorphisms in the non-coding regions were detected (compared to C. glabrata ATCC90030), no nonsynonymous mutation was detected in 35 fluconazole-resistant and three susceptible dose-dependent isolates. However, two nonsynonymous mutations (Y141H $+\mathrm{L} 381 \mathrm{M})$ were detected in one fluconazole-resistant isolate (Kw861/13). Isolate Kw861/13 was also sequenced earlier for ERG11 as part of another study and thus revealed the same mutations described in the previous study ${ }^{29}$. The ERG11 sequence variations observed in this study were used to study molecular epidemiology of fluconazole-resistance in Kuwait by combining ERG11 data with data from other loci. Concatenated sequence data comprising ERG11, ITS region of rDNA, extended hotspot-1 and hotspot-2 of FKS1 and FKS2 gene regions were aligned and the Neighbor-Joining phylogenetic tree is shown in Fig. 2. The data showed that 34 of 36 fluconazole-resistant C. glabrata isolates in Kuwait were unique strains while only two fluconazole-resistant isolates shared the same genotype.

\section{Discussion}

All 75 isolates, including 66 isolates collected during 2014-2017, used in this study were identified as C. glabrata sensu stricto by a combination of phenotypic and molecular methods. PCR-sequencing of ITS region of rDNA identified 23 different haplotypes among 51 isolates including 16 isolates with unique haplotypes. The data are consistent with a recent study showing that the ITS region of rDNA varies considerably among C. glabrata strains $^{33}$. None of the clinical isolate produced creamy white growth on CHROMagar Candida or yielded an amplicon of $\sim 299$ bp which is characteristic of C. bracarensis strains or an amplicon of $\sim 411$ bp which is characteristic of C. nivariensis strains in PCR assay ${ }^{33}$.

The AST data by Etest and BMD method identified 70 (93.3\%) isolates as susceptible and five isolates as resistant to micafungin. The data also showed that 70 (93.3\%) isolates exhibited excellent essential agreement as they yielded MIC values within 2-fold dilution difference by the two methods while only five isolates yielded poor essential agreement. However, the categorical agreement between the two methods was perfect as 70 and five isolates were scored as micafungin-susceptible and micafungin-resistant, respectively, by both methods. Espinel-Ingroff et al. ${ }^{34}$, reported an essential agreement of $95 \%$ and a categorical agreement of $97 \%$ between Etest and CLSI BMD method while Marcos-Zambrano et al..$^{35}$, reported an essential agreement of $90 \%$ and a categorical agreement of $>90 \%$ between Etest and EUCAST BMD method. Bougnoux et al. ${ }^{36}$, in a recent study based on 933 Candida species isolates (including 152 C. glabrata isolates) reported an essential agreement of $98.5 \%$ and a categorical agreement of $98.2 \%$ between Etest and EUCAST BMD method. Similar to these studies, our data also support that Etest is an easy and reliable method for routine AST of clinical C. glabrata isolates to micafungin.

Since FKS1 and FKS2 genes are homologous, a single amplification primer pair was carefully designed for PCR amplification of hotspot-1 regions of FKS1 and FKS2 genes but the sequence of both strands for each gene was obtained by using gene-specific sequencing primers as described under 'Materials and Methods'. Similarly, hotspot-2 regions of FKS1 and FKS2 were also amplified by using another common amplification primer pair and the amplicons were again sequenced by using gene-specific sequencing primers. This novel approach was highly efficient and cost effective as it reduced the work-load and material requirement for PCR amplification reactions and purification of amplicons by $50 \%$. 


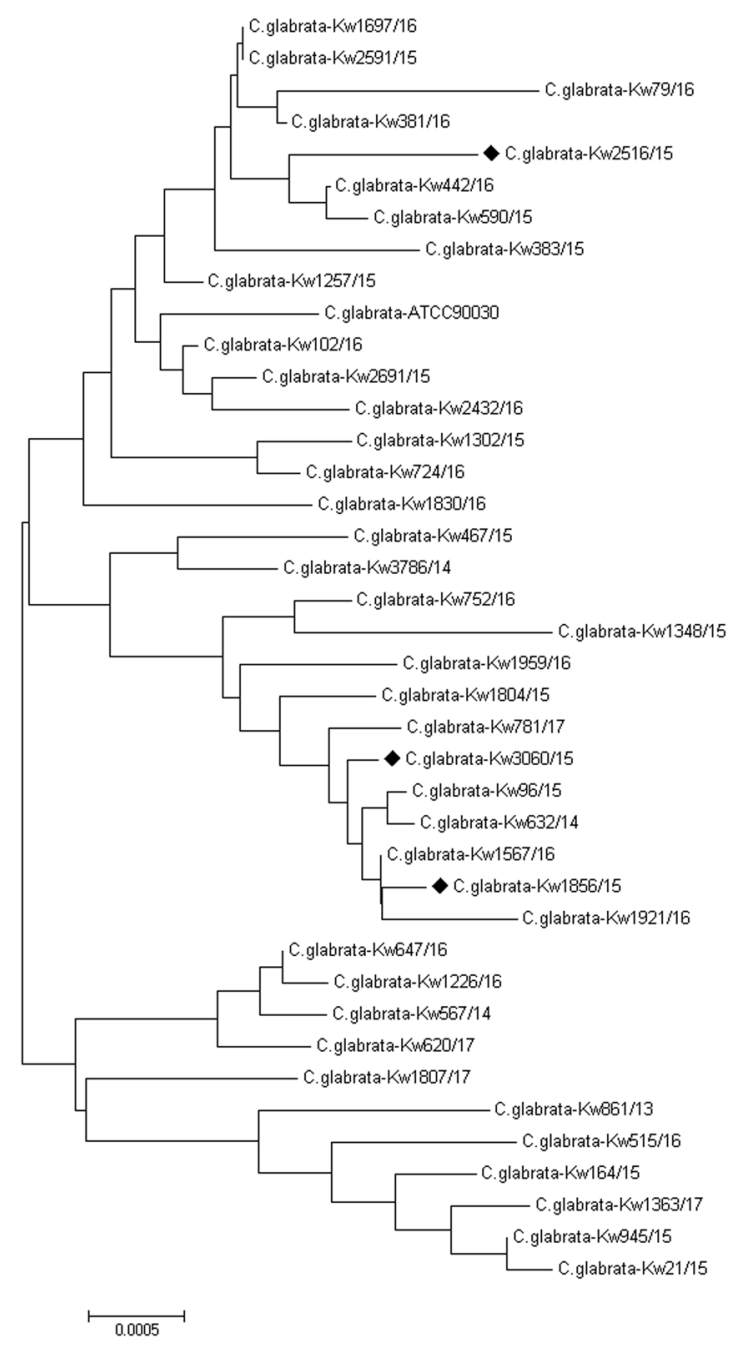

Figure 2. Neighbor-Joining phylogenetic tree based on Maximum Composite Likelihood of concatenated sequence of ERG11, ITS region of rDNA, and extended sequences of hotspot-1 and hotspot-2 of FKS1 and FKS2 genes for 36 fluconazole-resistant C. glabrata isolates. Three fluconazole-susceptible C. glabrata isolates $(\boldsymbol{\nabla})$, in addition to C. glabrata ATCC90030 reference strain were included for comparison purpose.

Although a few synonymous mutations were detected, the amino acid sequence data for hotspot-1 and hotspot-2 of FKS1 and hotspot-2 of FKS2 from all 75 isolates were identical (wild-type) to the sequence from reference C. glabrata strain ATCC90030. However, amino acid sequence data for hotspot-1 of FKS2 from only 70 isolates which were susceptible to micafungin were wild-type. Of the five micafungin-resistant isolates, four isolates contained a nonsynonymous (S663P) mutation and one isolate contained a three nucleotide deletion (corresponding to $\mathrm{F} 659 ; \Delta \mathrm{F} 659$ ) in hotspot-1 of FKS2. Thus all five micafungin-resistant isolates from Kuwait harbored a nonsynonymous or deletion mutation in hotspot-1 of FKS2 only. Our data are consistent with other reports showing that mutations in hotspot-1 of FKS2 occur more frequently (particularly at F659 and S663) than mutations in hotspot-1 of FKS1 in echinocandin-resistant C. glabrata isolates from diverse geographical locations with S663P mutation occurring more frequently ${ }^{14,22,37-41}$. Similar to another previous study ${ }^{37}$, all five micafungin-resistant C. glabrata isolates from Kuwait were also genotypically distinct strains.

Previous studies have shown that S663P and $\triangle \mathrm{F} 659$ mutations in hotspot-1 of FKS2 reduce echinocandin sensitivity in mutant 1,3- $\beta$-D-glucan synthase and the mutant enzyme exhibits reduced catalytic efficiency relative to wild-type enzyme ${ }^{42}$. Clinical significance of $\Delta \mathrm{F} 659$ mutation in hotspot-1 of FKS2 has also been shown in few studies. Lewis et al. ${ }^{32}$, reported clinical and microbiological failure in a patient with candidemia due to $C$. glabrata. The initial isolate before therapy was susceptible to micafungin and carried wild-type sequence for FKS genes while the isolate obtained after eight days of therapy was resistant to micafungin and contained $\Delta$ F659 mutation in hotspot- 1 of FKS2. Saraya et al. ${ }^{23}$, also reported a fatal case of fungemia in a patient due to C. glabrata. Resistance to micafungin developed during therapy and the resistant strain contained $\Delta F 659$ mutation in hotspot-1 of FKS2. Mutations in hotspot- 2 of FKS2 occur rarely while mutations in hotspot- 2 of FKS1, to the best of our knowledge, have not been reported so far ${ }^{14,22,37-42}$.

The history of previous exposure to echinocandins was available for four of five patients yielding micafungin-resistant C. glabrata isolates with mutations at F659 or S663 in hotspot-1 of FKS2. Three patients were 
females including two elderly patients ( $\geq 74$ years). All four patients had received caspofungin for 14 days as treatment or prophylaxis and C. glabrata strains were isolated from urine samples from all four patients. Whether $C$. glabrata was also recovered from invasive sites from these four patients was not known. Our results thus confirm previous findings that resistance to echinocandins and FKS mutations mainly arise in Candida species (including C. glabrata) as a result of previous exposure to these drugs which could be as short as $8-13$ days s $^{1422-24,37,39,43}$. The data also show that urinary tract provides a favorable niche for easy development of resistance not only to amphotericin $\mathrm{B}^{29}$ as was shown recently but also to echinocandins as reported in this study.

Three of five micafungin-resistant isolates were also resistant while two isolates exhibited intermediate resistance to caspofungin by Etest. Cross-resistance among echinocandins has been observed in several studies as they share the same mechanism of action ${ }^{19,20,42}$. In a recent study based on a global collection of invasive Candida species isolates collected over two decades (1997 to 2016), 2.2\%, 3.5\% and 1.7\% of C. glabrata isolates were resistant to anidulafungin, caspofungin and micafungin, respectively ${ }^{14}$. Surprisingly resistance to micafungin was not detected in C. glabrata isolates from Latin America, possibly reflecting the association of resistance development with specific genotypes ${ }^{14}$. As stated above, all five micafungin-resistant isolates (including two isolates with intermediate resistance to caspofungin) harbored a mutation in hotspot- 1 of $F K S 2$, however, a caspofungin-resistant isolate that was susceptible to micafungin contained wild-type FKS sequences in our study. An additional 22 isolates exhibited intermediate resistance to caspofungin but were susceptible to micafungin and contained wild-type sequences for $F K S$ genes. The $F K S$ mutations are now regarded as a better predictor of non-susceptibility of $C$. glabrata to echinocandins and poor response to treatment ${ }^{22-24,32,44-46}$. Taken together, our data suggest that micafungin can serve as an acceptable surrogate marker for the prediction of susceptibility or resistance of Candida species to caspofungin. Our results agree with data reported in few other studies. A previous study involving a large collection $(n=3674)$ of clinical isolates of eight Candida species concluded that micafungin serves as an acceptable surrogate marker for the prediction of susceptibility and resistance of Candida spp. to caspofungin ${ }^{47}$. Other studies have also made similar observations due to lack of reproducibility of caspofungin MIC test results or due to high degree of caspofungin MIC variability during both, intra- and interlaboratory testing ${ }^{48,49}$.

Since clinical Candida species isolates show wide variations in the MIC values for echinocandins, particularly caspofungin, it has been suggested to use epidemiological cutoff values (ECVs) instead of clinical break points to identify drug-resistant strains ${ }^{50,51}$. Although the number of C. glabrata isolates analyzed in this study is small compared to other studies that have used method-dependent ECVs to define C. glabrata isolates with reduced susceptibility to echinocandins, we also determined the ECVs for micafungin among our collection of C. glabrata isolates. Interestingly all 70 isolates lacking $F K S$ mutations yielded ECVs of $0.03 \mu \mathrm{g} / \mathrm{ml}$ by both Etest and BMD method to define wild-type organisms. Consistent with previously defined limits, the distribution of MIC values covered only two twofold dilution steps surrounding the modal MICs ${ }^{50-52}$. The data further support that FKS mutations are a better predictor of non-susceptibility of $C$. glabrata to echinocandins ${ }^{22-24,32,44-46}$.

Only one micafungin resistant C. glabrata isolate exhibited cross resistance to fluconazole and thus exhibited multidrug-resistant phenotype. On the other hand, all five micafungin resistant C. glabrata isolates were susceptible to amphotericin B. In the United States, the rate of resistance of C. glabrata to echinocandins has been increasing steadily and $\sim 9 \%$ of fluconazole-resistant bloodstream isolates were also resistant to echinocandins ${ }^{14,22,30,39}$. Emergence of resistance to echinocandins in C. glabrata and increasing reports of multidrug resistance to azoles, echinocandins and amphotericin B is a worrisome development as it severely limits the choice of antifungal drugs for the treatment of invasive C. glabrata infections s $^{13,14,20,30,31}$. In this context, multidrug resistance detection in only one C. glabrata isolate in Kuwait is encouraging, however, continued surveillance studies are needed to provide accurate estimates of trends in antifungal resistance and their impact on treatment outcome.

C. glabrata isolates analyzed in this study included four isolates that exhibited reduced susceptibility to amphotericin B that were analyzed recently for molecular resistance mechanisms ${ }^{29}$. One isolate (Kw861/13) cross-resistant to fluconazole contained two $(\mathrm{Y} 141 \mathrm{H}+\mathrm{L} 381 \mathrm{M})$ nonsynonymous mutations which abrogated the function of $E R G 11$, accumulated lanosterol and conferred resistance to fluconazole ${ }^{29}$. C. glabrata isolates analyzed in this study included 35 other strains with reduced susceptibility to fluconazole. Since non-synonymous mutations in ERG11 have rarely been reported in fluconazole-resistant C. glabrata ${ }^{29,53}$, all 36 fluconazole-resistant and three selected fluconazole-susceptible isolates were analyzed to see if additional fluconazole-resistant strains from Kuwait also contain nonsynonymous/nonsense mutations in ERG11.

PCR-sequencing of ERG11 did not detect any nonsynonymous/deletion mutation in the three susceptible dose-dependent isolates or the remaining 35 fluconazole-resistant isolates analyzed in this study. However, several synonymous mutations within the coding region of $E R G 11$ and/or insertion/deletion/single nucleotide polymorphisms in the non-coding regions were detected in many isolates. Since in addition to ERG11, DNA sequence data for hotspot-1 and hotspot-2 regions of FKS1 and FKS2 and ITS region of rDNA were also available for all 36 fluconazole-resistant C. glabrata isolates, concatenated sequence data were used for determining genetic relatedness among fluconazole-resistant C. glabrata isolates in Kuwait. The phylogenetic tree generated from the combined data sets showed that only two isolates clustered together while 34 isolates were genotypically distinct strains. Thus vast majority of fluconazole-resistant C. glabrata strains in Kuwait were genotypically distinct strains implying independent origin of fluconazole resistance development in our isolates. Molecular epidemiology of fluconazole-resistant C. glabrata strains has been rarely studied. One study showed that MALDI TOF MS data can be used to classify C. glabrata strains according to their fluconazole susceptibility profile ${ }^{54}$. Hou et $a l .{ }^{55}$, performed molecular fingerprinting of 411 C. glabrata isolates (including 68 fluconazole-resistant strains) from China by six-loci-based multilocus sequence typing (MLST) and six-polymorphic markers-based microsatellite typing (MT). Based on MLST, only 35 sequence types (STs) were identified among 273 C. glabrata isolates and most of the 68 fluconazole-resistant strains clustered into a single ST (ST7). Although MT analysis was more discriminatory as 79 genotypes were identified among 411 C. glabrata isolates, $125(30.4 \%)$ and 51 (12.4\%) 
isolates clustered in T25 and T31 types, respectively. Furthermore, T25 and T31 were also the predominant genotypes in fluconazole-resistant isolates ${ }^{55}$. Thus our multiple gene loci-based fingerprinting approach appears to be more discriminatory than MLST or MT analyses for molecular fingerprinting of fluconazole-resistant C. glabrata strains.

Our study has few limitations. The AST against fluconazole, caspofungin and amphotericin B was performed only by Etest and not by the reference BMD method. The molecular basis of resistance to fluconazole was investigated only by analysis of ERG11 while other resistance-conferring mechanisms such as sequence analyses of the transcription factor $P D R 1$ for gain-of-function mutations were not investigated due to lack of funds.

In conclusion, all 75 clinical isolates used in this study were identified as C. glabrata sensu stricto by a combination of phenotypic and molecular methods. Five of $75 \mathrm{C}$. glabrata isolates were resistant to micafungin by both, Etest and the reference BMD method. Only three of these five isolates were also resistant to caspofungin while two showed intermediate resistance. All micafungin-resistant isolates harbored a nonsynonymous or deletion mutation in hotspot-1 of FKS2 gene and were genotypically distinct strains. All micafungin-susceptible isolates were wild-type for hotspot-1 and hotspot-2 regions of FKS1 and FKS2 genes. Multidrug resistance (resistance to fluconazole and echinocandins) was observed in only one isolate. Micafungin clinical breakpoints by EUCAST were more reliable than CLSI breakpoints in discriminating echinocandin-resistant C. glabrata isolates from wild-type isolates and micafungin may also serve as a surrogate marker for predicting the susceptibility or resistance of C. glabrata to caspofungin. Amphotericin B resistance was detected in four of 75 (5\%) C. glabrata isolates while fluconazole resistance was detected in 36 of 75 (48\%) isolates. Only one C. glabrata isolate contained nonsynonymous mutations in ERG11. Molecular fingerprinting of fluconazole-resistant isolates showed that 34 isolates were unique strains suggesting that resistance development in C. glabrata to fluconazole in Kuwait is not clonal.

\section{Materials and Methods}

Yeast strains, growth conditions and phenotypic and molecular identification. Reference strains of C. glabrata (ATCC90030 and CBS138), C. albicans (ATCC90098), C. parapsilosis (ATCC22019) and C. krusei (ATCC6258) were used during this study. A total of 75 C. glabrata isolates originating from urogenital tract $(n=29)$, respiratory tract $(n=20)$, bloodstream $(n=12)$, ascitic/cavitary fluid $(n=3)$, wound $(n=3)$, skin $(\mathrm{n}=2)$ and other miscellaneous (peritoneal dialysis fluid, percutaneous endoscopic gastrostomy site, rectum, bed sore, pus, and from an unknown site) sites $(n=6)$ collected during 2007-2017 and maintained in the Fungal Culture Collection of Mycology Reference Laboratory (Department of Microbiology, Faculty of Medicine, Kuwait University) were used. The isolates were cultured from clinical specimens taken from patients after obtaining verbal consent as part of routine patient care and diagnostic work-up at nine different hospitals across Kuwait. The study did not involve direct contact with patients and the results are reported on deidentified samples without revealing patient identity. The study was approved by the Ethical Committee of Health Sciences Center, Kuwait University (approval letter VDR/EC/30 dated April 6, 2017) and all the methods and investigations were performed in accordance with their guidelines and regulations. The need for informed consent was waived by Health Sciences Center Ethical Committee. The blood specimens were cultured in Bact T/Alert Blood Culture System (BD Diagnostics, Sparks, MD) while other specimens were cultured on Sabouraud dextrose agar (SDA) supplemented with chloramphenicol $(50 \mathrm{mg} / \mathrm{L})$ as described previously ${ }^{56}$. The bloodstream isolates were also sub-cultured on SDA with/without additional supplements, as described previously ${ }^{28}$.

The isolates were initially identified as C. glabrata sensu lato by Vitek2 yeast identification system (bioMerieux, Marcy-lEtoile, France). All isolates were tested by growth on CHROMagar Candida (Becton Dickinson, Bootle, UK) for phenotypic identification and the results were interpreted according to manufacturer's instructions and as described previously ${ }^{57}$. The genomic DNA from the isolates was extracted by the rapid boiling method using Chelex-100 or by using Gentra Puregene Yeast DNA extraction kit (Qiagen, Hilden Germany) used according to kit instructions and as described previously ${ }^{58}$. Molecular identification was performed by PCR amplification of internal transcribed spacer (ITS) region of rDNA by using mCGLF, mCNIF, mCBRF and mCGCR primers and detection of amplicons by agarose gel electrophoresis, as described previously ${ }^{33}$. The identity of 51 selected isolates was also confirmed by sequencing of ITS region of rDNA by using panfungal primers, as described previously ${ }^{59}$. BLAST searches (http://blast.ncbi.nlm.nih.gov/Blast.cgi?) were performed and $>99 \%$ sequence identity was used for species identification ${ }^{60}$.

Antifungal susceptibility testing. The in vitro AST of C. glabrata isolates to fluconazole, amphotericin $B$, caspofungin and micafungin was performed by Etest (bioMérieux SA, Marcy-l'-Etoile, France) in accordance with the manufacturer's instructions and as described previously ${ }^{61}$. Reference strains of C. parapsilosis (ATCC22019) and C. albicans (ATCC90028) were used for quality control. The European Committee on Antimicrobial Susceptibility Testing (EUCAST) clinical breakpoints version 9.0 were followed to determine the susceptibility of the isolates as follows: fluconazole; $\leq 32 \mu \mathrm{g} / \mathrm{ml}$, susceptible dose dependent; $>64 \mu \mathrm{g} / \mathrm{ml}$, resistant; amphotericin B; $<1 \mu \mathrm{g} / \mathrm{ml}$, susceptible; $>1 \mu \mathrm{g} / \mathrm{ml}$, resistant; micafungin; $\leq 0.03 \mu \mathrm{g} / \mathrm{ml}$, susceptible; $>0.03 \mu \mathrm{g} / \mathrm{ml}$, resistant. However, the Clinical and Laboratory Standards Institute (CLSI) breakpoints were followed to determine the susceptibility of the isolates against caspofungin (EUCAST has not established breakpoints for Candida spp. due to high variability in MIC values) and were as follows: $\leq 0.12 \mu \mathrm{g} / \mathrm{ml}$, susceptible; $0.25 \mu \mathrm{g} / \mathrm{ml}$ to $<0.5 \mu \mathrm{g} /$ $\mathrm{ml}$, intermediate; $\geq 0.5 \mu \mathrm{g} / \mathrm{ml}$, resistant. Quality control was ensured by testing C. krusei (ATCC6258) as recommended by EUCAST ${ }^{62-64}$.

The AST of C. glabrata isolates to micafungin was also determined by reference BMD method in 96-well tissue culture plate by following the protocol described in the EUCAST Definitive document 7.3.1. The MIC was determined as the drug concentration that yielded $\geq 50 \%$ growth inhibition compared to drug-free control and C. krusei (ATCC6258) was used as quality control ${ }^{62}$. 
PCR-sequencing of hotspot-1 and hotspot- 2 regions of FKS1 and FKS2 genes. The hotspot-1 of FKS1 and FKS2 genes was amplified by using a common forward (CgFKS-1F, 5'-ATGCCATTRGGTGGTCTK TTCAC-3') and reverse (CgFKS-1R, 5'-ATRGCAAGYAAATGTTCTCTGTACA-3) primer pair. Similarly, hotspot-2 of both FKS1 and FKS2 genes was amplified by using another common forward (CgFKS-2F, 5'-GTGAACAAATGTTGTCCCGTGA-3') and reverse (CgFKS-2R, 5'-GCAAATCTGGAGTAYAAAATKGAGA $\left.-3^{\prime}\right)$ primer pair. Other PCR reaction and cycling conditions were same as described previously ${ }^{24}$. PCR amplicons were purified and both strands were sequenced for hotspot-1 and hotspot-2 of FKS1 and FKS2. Sequencing reactions with amplicons obtained with CgFKS-1F + CgFKS-1R primers for hotspot-1 of FKS1 were carried out by using CgFKS1-1FS (5'-AAAGTCTACCAGACGTTACGTC-3') or CgFKS1-1RS (5'-GGAGTCAAAATAGAAATACCCAAG-3') primer and for hotspot-1 of FKS2 by using CgFKS2-1FS (5'-CAAAAATCAAGTAGAAGATATGTT-3') or CgFKS2-1RS (5'-AGGAGTTAAGATGGAAATACCTAGA -3') primer. Similarly, sequencing reactions for hotspot-2 of FKS1 were carried out by using CgFKS1-2FS (5'-AGGTACACAACTTCCAATTGA-3') or CgFKS1-2RS (5'-AATCGCTCAACAAAGCAGATGAGT-3') primer and for hotspot-2 of FKS2 by using CgFKS2-2FS (5'-AGGTACACAATTGCCCGTAGA-3') or CgFKS22RS (5'-TGTCACTCAATAGAGCAGCAGAA-3') primer. Sequencing reactions were performed and processed as described previously ${ }^{24}$. Sequence data for hotspot-1 and hotspot-2 regions of FKS1 and FKS2 were compared with corresponding sequences from reference C. glabrata strain ATCC90030 by using Clustal Omega (https:// www.ebi.ac.uk/Tools/msa/clustalo/).

PCR-sequencing of ERG11 gene. The ERG11 gene was amplified as two overlapping fragments by using C. glabrata ATCC 90030 as reference. The N-terminal fragment was amplified by using CgERG11F (5'-TCCACCTCGAACCCGTATA-3') and CgERG11RS3 (5'-ATCAAGACACCAATCAATAGGTT-3') primers while C-terminal fragment was amplified by using CgERG11FS3 (5'-GACGTGAGAAGAACGATATCCA-3') and CgERG11R (5'-TCCATGTTGATATTCACGATGACT-3') primers. Other PCR reaction and cycling conditions were same as described previously ${ }^{28,29}$. N-terminal amplicons were sequenced with CgERG11FS1 (5'-GAACCCGTATACTCATCTCGTA-3'), CgERG11FS2 (5'-GGTGATATCTTCTCTTTCATGCTA-3' ), CgERG11RS3 (5'- AGTAAGCAGCTTCAGCGGAAACA-3') and CgERG11RS4 (5'-ATCAAGACACC AATCAATAGGTT-3') primers. C-terminal amplicons were sequenced with CgERG11FS3 (5'-GACGTGAGAAG AACGATATCCA-3'), CgERG11FS4 (5'-GTTACACTCACTTGCAAGAAGAA-3'), CgERG11RS1 (5'-CACGATGACTTACTATTAGGCTAA-3') and CgERG11RS2 (5'-CGAAACCGTAATCAACTTCGTCA-3') primers. Sequencing reactions were performed and processed as described previously ${ }^{28,29}$. Nucleotide and amino acid sequences were compared with wild-type sequence from C. glabrata ATCC90030 using Clustal Omega (http://www.ebi.ac.uk/Tools/msa/clustalo/).

The DNA sequence data have been submitted to GenBank/EMBL/DDBJ databases under accession numbers LR757901 to LR757940.

Molecular fingerprinting of micafungin-resistant C. glabrata isolates. The phylogenetic relationship among micafungin-resistant and some randomly selected micafungin-susceptible $C$. glabrata isolates was also studied. The DNA sequence data for ITS region of rDNA together with hotspot- 1 and hotspot- 2 of FKS1 and hotspot-1 and hotspot-2 of FKS2 were concatenated and the combined data set were used to construct Neighbor-Joining phylogenetic tree using Maximum Composite Likelihood settings by using Molecular Evolutionary Genetics Analysis Version 7.0 (MEGA7) software (http://www.megasoftware.net/mega.php). The robustness of tree branches was assessed by bootstrap analysis of 1,000 replicates. The isolates were considered belonging to the same genotype when they contained the same sequence for all loci.

Molecular fingerprinting of fluconazole-resistant C. glabrata isolates. The phylogenetic relationship among fluconazole-resistant and some randomly selected fluconazole-susceptible C. glabrata isolates was studied by constructing dendrograms based on DNA sequence data for ERG11 alone or in combination with ITS region of rDNA and hotspot-1 and hotspot-2 of FKS1 and FKS2 genes. The sequences were concatenated and the combined sequence data set were used to construct phylogenetic tree and the data were interpreted as described above.

Received: 3 February 2020; Accepted: 17 March 2020;

Published online: 10 April 2020

\section{References}

1. Diekema, D., Arbefeville, S., Boyken, L., Kroeger, J. \& Pfaller, M. The changing epidemiology of healthcare-associated candidemia over three decades. Diagn. Microbiol. Infect. Dis. 73, 45-48 (2012).

2. Guinea, J. Global trends in the distribution of Candida species causing candidemia. Clin. Microbiol. Infect. 20(Supp. 6), 5-10 (2014).

3. Pappas, P. G. et al. Clinical practice guideline for the management of candidiasis: 2016 update by the Infectious Diseases Society of America. Clin. Infect. Dis. 62, e1-e50 (2016).

4. Khan, Z., Ahmad, S., Joseph, L. \& Chandy, R. Candida dubliniensis: an appraisal of its clinical significance as a bloodstream pathogen. PLoS One 7, e32952 (2012).

5. Sanguinetti, M., Posteraro, B. \& Lass-Flörl, C. Antifungal drug resistance among Candida species: mechanisms and clinical impact. Mycoses 58 Suppl 2, 2-13 (2015).

6. Pfaller, M. A. et al. Epidemiology and outcomes of invasive candidiasis due to non-albicans species of Candida in 2,496 patients: data from the Prospective Antifungal Therapy (PATH) registry 2004-2008. PLoS One 9, e101510 (2014).

7. Andes, D. R. et al. The epidemiology and outcomes of invasive Candida infections among organ transplant recipients in the United States: results of the Transplant-Associated Infection Surveillance Network (TRANSNET). Transpl. Infect. Dis. 18, 921-931 (2016). 
8. Ahmad, S., Khan, Z., Al-Sweih, N., Alfouzan, W. \& Joseph, L. Candida auris in various hospitals across Kuwait and their susceptibility and molecular basis of resistance to antifungal drugs. Mycoses 63, 104-112 (2020).

9. Khan, Z. et al. Invasive Candida auris infections in Kuwait hospitals: epidemiology, antifungal treatment and outcome. Infection 46, 641-650 (2018).

10. Papon, N., Courdavault, V., Clastre, M. \& Bennett, R. J. Emerging and emerged pathogenic Candida species: beyond the Candida albicans paradigm. PLoS Pathog. 9, e1003550 (2013).

11. Arendrup, M. C. et al. ESCMID and ECMM joint clinical guidelines for the diagnosis and management of rare invasive yeast infections. Clin. Microbiol. Infect. 20 Suppl 3, 76-98 (2014).

12. Ostrosky-Zeichner, L. Candida glabrata and FKS mutations: witnessing the emergence of the true multidrug-resistant. Candida. Clin. Infect. Dis. 56, 1733-1734 (2013).

13. Colombo, A. L., Júnior, J. N. A. \& Guinea, J. Emerging multidrug-resistant Candida species. Curr. Opin. Infect. Dis. 30, 528-538 (2017).

14. Pfaller, M. A., Diekema, D. J., Turnidge, J. D., Castanheira, M. \& Jones, R. N. Twenty Years of the SENTRY Antifungal Surveillance Program: Results for Candida Species from 1997-2016. Open Forum Infect. Dis. 6(Suppl 1), S79-S94 (2019).

15. Pfaller, M. A., Moet, G. J., Messer, S. A., Jones, R. N. \& Castanheira, M. Candida bloodstream infections: comparison of species distributions and antifungal resistance patterns in community-onset and nosocomial isolates in the SENTRY Antimicrobial Surveillance Program, 2008-2009. Antimicrob. Agents Chemother. 55, 561-566 (2011).

16. Lockhart, S. R. et al. Species identification and antifungal susceptibility testing of Candida bloodstream isolates from populationbased surveillance studies in two U.S. cities from 2008 to 2011. J. Clin. Microbiol. 50, 3435-3442 (2012).

17. Cleveland, A. A. et al. Changes in incidence and antifungal drug resistance in candidemia: results from population-based laboratory surveillance in Atlanta and Baltimore, 2008-2011. Clin. Infect. Dis. 55, 1352-1361 (2012).

18. Colombo, A. L. et al. Prognostic factors and historical trends in the epidemiology of candidemia in critically ill patients: an analysis of five multicenter studies sequentially conducted over a 9-year period. Intensive Care Med. 40, 1489-1498 (2014).

19. Pfaller, M. A. Antifungal drug resistance: mechanisms, epidemiology, and consequences for treatment. Am. J. Med. 125, S3-S13 (2012).

20. Kołaczkowska, A. \& Kołaczkowski, M. Drug resistance mechanisms and their regulation in non-albicans Candida species. J. Antimicrob. Chemother. 71, 1438-1450 (2016).

21. Dannaoui, E. et al. Candida spp. with acquired echinocandin resistance, France, 2004-2010. Emerg. Infect. Dis. 8, 86-90 (2012).

22. Alexander, B. D. et al. Increasing echinocandin resistance in Candida glabrata: clinical failure correlates with presence of $F K S$ mutations and elevated minimum inhibitory concentrations. Clin. Infect. Dis. 56, 1724-1732 (2013).

23. Saraya, T. et al. Breakthrough invasive Candida glabrata in patients on micafungin: a novel FKS gene conversion correlated with sequential elevation of MIC. J. Clin. Microbiol. 52, 2709-2712 (2014).

24. Khan, Z. et al. Development of echinocandin resistance in Candida tropicalis following short-term exposure to caspofungin for empiric therapy. Antimicrob. Agents Chemother. 62, e01926-17 (2018).

25. Khan, Z. U. et al. Emergence of resistance to amphotericin B and triazoles in Candida glabrata vaginal isolates in a case of recurrent vaginitis. J. Chemother. 20, 488-491 (2008).

26. Vandeputte, P. et al. A nonsense mutation in the ERG6 gene leads to reduced susceptibility to polyenes in a clinical isolate of Candida glabrata. Antimicrob. Agents Chemother. 52, 3701-3709 (2008).

27. Hull, C. M. et al. Two clinical isolates of Candida glabrata exhibiting reduced sensitivity to amphotericin B both harbor mutations in ERG2. Antimicrob. Agents Chemother. 56, 6417-6421 (2012).

28. Khan, Z., Ahmad, S., Joseph, L. \& Al-Obaid, K. Isolation of cholesterol-dependent, multidrug-resistant Candida glabrata strains from blood cultures of a candidemia patient in Kuwait. BMC Infect. Dis. 14, 188 (2014).

29. Ahmad, S. et al. ERG6 and ERG2 are major targets conferring reduced susceptibility to amphotericin B in clinical Candida glabrata isolates in Kuwait. Antimicrob. Agents Chemother. 63, e01900-18 (2019).

30. Healey, K. R. et al. Prevalent mutator genotype identified in fungal pathogen Candida glabrata promotes multi-drug resistance. Nat. Commun. 7, 1-10 (2016).

31. Healey, K. R. \& Perlin, D. S. Fungal resistance to echinocandins and the MDR phenomenon in Candida glabrata. J. fungi 4, 1-14 (2018).

32. Lewis et al. Rapid emergence of echinocandin resistance in Candida glabrata resulting in clinical and microbiologic failure. Antimicrob. Agents Chemother. 57, 4559-4561 (2013).

33. Asadzadeh, M., Alanazi, A. F., Ahmad, S., Al-Sweih, N. \& Khan, Z. Lack of detection of Candida nivariensis and Candida bracarensis among 440 clinical Candida glabrata sensu lato isolates in Kuwait using a newly developed PCR assay targeting the ITS region of rDNA. PLoS One 14, e0223920 (2019).

34. Espinel-Ingroff, A., Cantón, E., Pelaez, T. \& Pemán, J. Comparison of micafungin MICs as determined by the Clinical and Laboratory Standards Institute broth microdilution method (M27-A3 document) and Etest for Candida spp. isolates. Diagn. Microbiol. Infect. Dis. 70, 54-59 (2011)

35. Marcos-Zambrano, L. J. et al. Comparison between the EUCAST procedure and the Etest for determination of the susceptibility of Candida species isolates to micafungin. Antimicrob. Agents Chemother. 57, 5767-5770 (2013).

36. Bougnoux, M. E. et al. Multicenter comparison of the Etest and EUCAST methods for antifungal susceptibility testing of Candida isolates to micafungin. Antimicrob. Agents Chemother. 60, 5088-5091 (2016).

37. Zimbeck, A. J. et al. FKS mutations and elevated echinocandin MIC values among Candida glabrata isolates from U.S. populationbased surveillance. Antimicrob. Agents Chemother. 54, 5042-5047 (2010).

38. Castanheira, M. et al. Frequency of FKS mutations among candida glabrata isolates from a 10-Year global collection of bloodstream infection isolates. Antimicrob. Agents Chemother. 58, 577-580 (2014).

39. Pham, C. D. et al. Role of FKS mutations in Candida glabrata: MIC values, echinocandin resistance, and multidrug resistance. Antimicrob. Agents Chemother. 58, 4690-4696 (2014).

40. Dudiuk, C. et al. Set of classical PCRs for detection of mutations in Candida glabrata FKS genes linked with echinocandin resistance. J. Clin. Microbiol. 52, 2609-2614 (2014).

41. Katiyar, S. et al. Evaluation of Polymorphic Locus Sequence Typing for Candida glabrata Epidemiology. J. Clin. Microbiol. 54, 1042-1050 (2016)

42. Garcia-Effron, G., Lee, S., Park, S., Cleary, J. D. \& Perlin, D. S. Effect of Candida glabrata FKS1 and FKS2 mutations on echinocandin sensitivity and kinetics of 1,3- $\beta$-D-glucan synthase: Implication for the existing susceptibility breakpoint. Antimicrob. Agents Chemother. 53, 3690-3699 (2009).

43. Shields, R. K. et al. The presence of an FKS mutation rather than MIC is an independent risk factor for failure of echinocandin therapy among patients with invasive candidiasis due to Candida glabrata. Antimicrob. Agents Chemother. 56, 4862-4869 (2012).

44. Wiederhold, N. P. Echinocandin resistance in Candida species: a review of recent developments. Curr. Infect. Dis. Rep. 18, 42 (2016).

45. Zhao, Y. et al. Rapid detection of FKS-associated echinocandin resistance in Candida glabrata. Antimicrob. Agents Chemother. 60, 6573-6577 (2016)

46. Bienvenu, A. L., Leboucher, G. \& Picot, S. Comparison of $f k s$ gene mutations and minimum inhibitory concentrations for the detection of Candida glabrata resistance to micafungin: A systematic review and meta-analysis. Mycoses 62, 835-846 (2019). 
47. Pfaller, M. A., Messer, S. A., Diekema, D. J., Jones, R. N. \& Castanheira, M. Use of micafungin as a surrogate marker to predict susceptibility and resistance to caspofungin among 3,764 clinical isolates of Candida by use of CLSI methods and interpretive criteria. J. Clin. Microbiol. 52, 108-114 (2014).

48. Arendrup, M. C. et al. Caspofungin Etest susceptibility testing of Candida species: Risk of misclassification of susceptible isolates of C. glabrata and C. krusei when adopting the revised CLSI caspofungin breakpoints. Antimicrob. Agents Chemother. 56, 3965-3968 (2012).

49. Espinel-Ingroff, A. et al. Interlaboratory variability of caspofungin MICs for Candida spp. using CLSI and EUCAST methods: should the clinical laboratory be testing this agent? Antimicrob. Agents Chemother. 57, 5836-5842 (2013).

50. Pfaller, M. A., Messer, S. A., Woosley, L. N., Jones, R. N. \& Castanheira, M. Echinocandin and triazole antifungal susceptibility profiles for clinical opportunistic yeast and mold isolates collected from 2010 to 2011: application of new CLSI clinical breakpoints and epidemiological cutoff values for characterization of geographic and temporal trends of antifungal resistance. J. Clin. Microbiol. 51, 2571-2581 (2013)

51. Espinel-Ingroff, A. et al. Multicenter study of method-dependent epidemiological cutoff values for detection of resistance in Candida spp. and Aspergillus spp. to amphotericin B and echinocandins for the Etest agar diffusion method. Antimicrob. Agents Chemother. 61, e01792-16 (2016).

52. Pfaller, M. A. et al. Wild-type MIC distributions, epidemiological cutoff values and species-specific clinical breakpoints for fluconazole and Candida: time for harmonization of CLSI and EUCAST broth microdilution methods. Drug Resist. Updat. 13, 180-195 (2010).

53. Hull, C. M. et al. Facultative sterol uptake in an ergosterol-deficient clinical isolate of Candida glabrata harboring a missense mutation in ERG11 and exhibiting cross-resistance to azoles and amphotericin B. Antimicrob. Agents Chemother. 56, 4223-4232 (2012).

54. Dhieb, C. et al. MALDI-TOF typing highlights geographical and fluconazole resistance clusters in Candida glabrata. Med. Mycol. 53, $462-469$ (2015).

55. Hou, X. et al. Molecular epidemiology and antifungal susceptibility of Candida glabrata in China (August 2009 to July 2014): a multi-center study. Front. Microbiol. 8, 880 (2017).

56. Khan, Z. et al. Changing trends in epidemiology and antifungal susceptibility patterns of six bloodstream Candida species isolates over a 12 -year period in Kuwait. PLoS One 14, e216250 (2019).

57. Lockhart, S. R. et al. Identification of Candida nivariensis and Candida bracarensis in a large global collection of Candida glabrata isolates: comparison to the literature. J. Clin. Microbiol. 47, 1216-1217 (2009).

58. Asadzadeh, M. et al. Simple, low-cost detection of Candida parapsilosis complex isolates and molecular fingerprinting of Candida orthopsilosis strains in Kuwait by ITS region sequencing and amplified fragment length polymorphism analysis. PLoS One 10, e0142880 (2015).

59. Khan, Z. U. et al. Cryptococcus randhawai sp. nov., a novel anamorphic basidiomycetous yeast isolated from tree trunk hollow of Ficus religiosa (peepal tree) from New Delhi, India. Antonie Van Leeuwenhoek 97, 253-259 (2010).

60. Schoch, C. L. et al. Nuclear ribosomal internal transcribed spacer (ITS) region as a universal DNA barcode marker for fungi. Proc. Natl. Acad. Sci. USA 109, 6241-6246 (2012).

61. Asadzadeh, M., Al-Sweih, N. A., Ahmad, S. \& Khan, Z. U. Antifungal susceptibility of clinical Candida parapsilosis isolates in Kuwait. Mycoses 51, 318-323 (2008).

62. European Committee on Antimicrobial Susceptibility Testing (EUCAST). EUCAST method for susceptibility testing of yeasts. EUCAST definitive document EDef 7.3.1. EUCAST. Vaxjo, Sweden (2017).

63. Clinical and Laboratory Standards Institute. Fourth informational supplement M27-S4. 4th. Wayne, PA: 2012. Reference method for broth dilution antifungal susceptibility testing of yeasts. Clinical Laboratory Standards Institute, Wayne, PA (2012).

64. Bourgeois, N. et al. Assessment of caspofungin susceptibility of Candida glabrata by the Etest, CLSI, and EUCAST methods, and detection of FKS1 and FKS2 mutations. Eur. J. Clin. Microbiol. Infect. Dis. 33, 247-252 (2014).

\section{Acknowledgements}

We thank Mrs. Leena Joseph and Dr. Mohammad Asadzadeh for technical support. This study was supported by College of Graduate Studies and Kuwait University Research Sector Grant No. YM08/17.

\section{Author contributions}

Conceived and designed the experiments: Z.A.B., S.A., and Z.K. Performed the experiments: Z.A.B. Analyzed the data: Z.A.B., S.A., and Z.K. Contributed reagents/materials/analysis tools: S.A., and Z.K. Wrote the paper: Z.A.B., S.A., and Z.K.

\section{Competing interests}

The authors declare no competing interests.

\section{Additional information}

Supplementary information is available for this paper at https://doi.org/10.1038/s41598-020-63240-z.

Correspondence and requests for materials should be addressed to S.A.

Reprints and permissions information is available at www.nature.com/reprints.

Publisher's note Springer Nature remains neutral with regard to jurisdictional claims in published maps and institutional affiliations.

Open Access This article is licensed under a Creative Commons Attribution 4.0 International

License, which permits use, sharing, adaptation, distribution and reproduction in any medium or format, as long as you give appropriate credit to the original author(s) and the source, provide a link to the Creative Commons license, and indicate if changes were made. The images or other third party material in this article are included in the article's Creative Commons license, unless indicated otherwise in a credit line to the material. If material is not included in the article's Creative Commons license and your intended use is not permitted by statutory regulation or exceeds the permitted use, you will need to obtain permission directly from the copyright holder. To view a copy of this license, visit http://creativecommons.org/licenses/by/4.0/.

(C) The Author(s) 2020 